\title{
Clostridium chauvoei, an Evolutionary Dead-End Pathogen
}

\author{
Lorenz Rychener ${ }^{1 \dagger}$, Saria In-Albon ${ }^{1 \dagger}$, Steven P. Djordjevic ${ }^{2}$, Piklu Roy Chowdhury², \\ Pamela Nicholson ${ }^{1}$, Rosangela E. Ziech ${ }^{3}$, Agueda C. de Vargas ${ }^{3}$, Joachim Frey ${ }^{1 *}$ and \\ Laurent Falquet ${ }^{4}$
}

${ }^{1}$ Institute of Veterinary Bacteriology, Vetsuisse Faculty, University of Bern, Bern, Switzerland, ${ }^{2}$ The iThree Institute, University of Technology Sydney, Ultimo, NSW, Australia, ${ }^{3}$ Department of Preventive Veterinary Medicine, Federal University of Santa Maria, Santa Maria, Brazil, ${ }^{4}$ Department of Biology, Swiss Institute of Bioinformatics, University of Fribourg, Fribourg, Switzerland

\section{OPEN ACCESS}

Edited by:

Jorge Blanco,

Universidade de Santiago

de Compostela, Spain

Reviewed by: Michel Popoff,

Institut Pasteur, France

María de Toro,

Centro de Investigación Biomédica de La Rioja (CIBIR), Spain

*Correspondence:

Joachim Frey

joachim.frey@vetsuisse.unibe.ch

${ }^{\dagger}$ Shared first authorship

Specialty section:

This article was submitted to

Infectious Diseases,

a section of the journal

Frontiers in Microbiology

Received: 01 May 2017

Accepted: 26 May 2017

Published: 09 June 2017

Citation:

Rychener L, In-Albon S, Djordjevic SP, Chowdhury PR,

Nicholson P, Ziech RE,

de Vargas AC, Frey $J$ and Falquet $L$

(2017) Clostridium chauvoei, an

Evolutionary Dead-End Pathogen.

Front. Microbiol. 8:1054.

doi: 10.3389/fmicb.2017.01054
Full genome sequences of 20 strains of Clostridium chauvoei, the etiological agent of blackleg of cattle and sheep, isolated from four different continents over a period of 64 years (1951-2015) were determined and analyzed. The study reveals that the genome of the species $C$. chauvoei is highly homogeneous compared to the closely related species $C$. perfringens, a widespread pathogen that affects human and many animal species. Analysis of the CRISPR locus is sufficient to differentiate most C. chauvoei strains and is the most heterogenous region in the genome, containing in total 187 different spacer elements that are distributed as $30-77$ copies in the various strains. Some genetic differences are found in the 3 allelic variants of fliC1, fliC2 and fliC3 genes that encode structural flagellin proteins, and certain strains do only contain one or two alleles. However, the major virulence genes including the highly toxic $\underline{C}$.

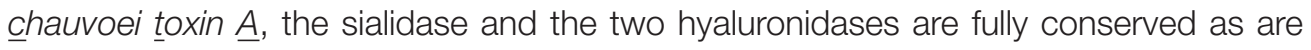
the metabolic and structural genes of $C$. chauvoei. These data indicate that $C$. chauvoei is a strict ruminant-associated pathogen that has reached a dead end in its evolution.

Keywords: Clostridium chauvoei, CRISPR, virulence genes, flagellin genes, blackleg, dead-end evolution

\section{INTRODUCTION}

Clostridium chauvoei, a Gram positive, highly pathogenic, strict anaerobic bacterium that is able to sporulate, is the etiologic agent of blackleg, a severe disease specifically of cattle and to a lesser extent also of small ruminants. Blackleg spreads globally manifesting as a fulminant myonecrosis that generally leads to the death of the animal within a short time. Due to the high mortality blackleg causes significant losses in livestock production (Hatheway, 1990; Groseth et al., 2011; Frey and Falquet, 2015). Pathological lesions of blackleg are mostly found in the muscular tissue of animals, often in leg muscles, from where the pathogen is commonly isolated. Animals generally get infected from C. chauvoei spores that contaminate the soil of pastures either from perished animals or via manure (Bagge et al., 2009; Lange et al., 2010). In cattle, the pathogen is taken up via the digestive tract or the respiratory tract from where C. chauvoei migrates to the muscle tissues where the spores remain dormant until specific conditions are generated, as tissular devitalization that promotes anaerobiosis, resulting in their germination, multiplication and consequently production of the exotoxins (Jubb et al., 1991). In small ruminants, skin lesions are also considered as a port of entry of the pathogen. Blackleg occurs with increased incidence during dry seasons that followed flooding, when the animals have to graze short plants and are closer in contact with their nostrils and muzzles to soil where the pathogen spreads during the preceding flooding (Hatheway, 1990; 
Useh et al., 2006a). C. chauvoei is considered as one of the most pathogenic Clostridium species. The infection by C. chauvoei causes myonecrosis, oedemic lesions and fever that is rapidly followed by lameness and death. Due to the rapid death of the animals, which mostly is the only sign of the disease, antibiotics are generally not used to cure blackleg. However, commercial and locally produced vaccines successfully control blackleg in ruminants (Uzal, 2012; Frey and Falquet, 2015). Blackleg is not notified as a zoonotic disease, although two isolated cases of human infections with $C$. chauvoei leading to lethal fulminant gas gangrene and neutropenic enterocolitis have been reported (Nagano et al., 2008; Weatherhead and Tweardy, 2012).

Current knowledge on pathogenicity of C. chauvoei reveals that toxins, a highly active DNAse, hyaluronidase, sialidase, and flagella represent the main virulence factors (Useh et al., 2003, 2006b; Vilei et al., 2011; Frey et al., 2012; Frey and Falquet, 2015). Among the postulated toxins, $\underline{C}$. chauvoei toxin A (CctA), a member of the $\beta$-barrel pore forming leukocidin superfamily, was shown to represent the main cytotoxic- and haemolytic activity of $C$. chauvoei. Recombinant purified CctA toxin produced in Escherichia coli is oxygen stable with regard to its haemolytic and cytotoxic activity. Guinea pigs vaccinated with a purified inactivated recombinant chimeric protein consisting of the CctA toxin and the E. coli heat labile toxin subunit B (LTB) as an adjuvant were protected against challenge with virulent $C$. chauvoei spores, revealing the central role of CctA in virulence (Frey et al., 2012). Besides toxin CctA, two further virulence factors, sialidase $\mathrm{NanA}$ and hyaluronidase $\mathrm{NagH}$ have been analyzed biochemically and genetically. The enzymes seem to enable C. chauvoei to stir from the initial site of infection which are generally believed to be oral or the respiratory tract or occasionally skin lesions, and get access to muscular tissue where the bacterium can replicate and cause myonecrosis, the typical pathological symptoms of blackleg (Vilei et al., 2011; Frey and Falquet, 2015).

Vaccines against blackleg consist of chemically inactivated bacteria of mostly relatively ancient strains providing outer membrane proteins and flagellar proteins that have been proposed as immunogens and bacterial culture supernatants that are expected to contain the main toxins (Mattar et al., 2002; Uzal, 2012). Recently it has been shown that the CctA toxin alone, which is conserved between C. chauvoei strains isolated worldwide, prepared by recombinant gene technology, provided full protection in a guinea pig infection model which serves as biological test for potency tests in the batch release procedure of commercial vaccines (Frey et al., 2012). Flagellar antigens are traditionally considered to be important virulence attributes and protective factors in vaccines (Chandler and Gulasekharam, 1974; Tamura and Tanaka, 1987; Tanaka et al., 1987; Tamura et al., 1995). They have been used recently as diagnostic tools for the serological detection of C. chauvoei infections and were shown to be antigenically highly conserved between the type strain ATCC10092 and strains isolated in India between 2010 and 2012 (Usharani et al., 2015).

Due to the short duration of infection of the bacterium in the animal host, during which the bacterium replicates extensively and forms spores that can survive for several years on pastures, C. chauvoei has a bi-modal lifestyle, similar to B. anthracis. Although it is generally known that pastures contaminated with spores of $C$. chauvoei represent the main epidemiological hazard for infection of cattle (Wolf et al., 2017), and that vaccination is the most efficient way to stop the epidemic, there is no knowledge of the phylogeny or molecular epidemiology of C. chauvoei.

In order to get insight into the molecular genetic evolution of C. chauvoei, to provide new tools for molecular epidemiological assessments, and to provide a scientific basis for the development of both novel vaccines as well as potency test that is independent of live animal infections, we have fully sequenced, analyzed and compared the genomes of 20 strains of $C$. chauvoei that were isolated between 1951 and 2015 and originated from Africa, Australia, Northern and Southern America and Europe. These strains originate mostly from internal strain repositories as there are only very few strains at public collections. Particular focus was given to the genetic variability of known and potential virulence genes such as the flagellar genes that provide motility.

\section{MATERIALS AND METHODS}

\section{Bacterial Strains}

Bacterial strains used in this study are listed in Table 1. They were sourced from a collection maintained at the Institute of Veterinary Bacteriology, University of Bern and originate either from own isolations from local blackleg cases, or were obtained during the last decades from various institutions worldwide for the purpose of reference species identifications or were ordered from international strain collections.

\section{Update of Reference Strain JF4335}

The genome of C. chauvoei strain JF4335 was sequenced with Pacific Bioscience RSII. After circularization, the sequence was corrected with Illumina HiSeq reads for the substitutions due to sequencing errors and for the frameshifts. The resulting full genome was annotated using the Prokka pipeline (Seemann, 2014) using annotation from the previous draft genome JF4335 (GenBank: CBML000000000.1) (Falquet et al., 2013) and C. perfringens ATCC 13124 (Genbank: NC_008261.1) and UniProtKB as additional database.

\section{Whole Genome Sequencing and SNVs Discovery}

The DNAs of the $20 \mathrm{C}$. chauvoei strains were subjected to sequencing by Illumina HiSeq using the Nextera XT DNA protocol for the library preparation and the V3 chemistry to produce 2 × 250 bp paired-end reads (Fasteris SA, Plan-lesOuates, Switzerland). The number of reads after filtering ranges from 0.6 to 4 million paired-end reads. All reads are available on the Sequence Read Archive (SRA: SRR5429428 - SRR5429447) and strain number and SRA accession number are linked in Table 1. The full genome of C. chauvoei strain JF4335 (LT799839), obtained as described above, was used as a reference. Illumina paired-end reads from all strains were mapped to the reference 
TABLE 1 | List of all strains included in the study with strain name, year of isolation, location of isolation, host animal, strain collection, reference and SNVs detected during the analysis.

\begin{tabular}{|c|c|c|c|c|c|c|c|}
\hline Strain nr. & Year of isolation & Origin & Animal host & Original strain collection / nr & Reference & SNVs & SRA Accession \\
\hline JF1863 & Unknown & Unknown & Bovine & ATCC 10092 ${ }^{\top}$ Type strain & & 134 & SRR5429447 \\
\hline JF2696 & 2002 & Brazil & Bovine & Vetsuisse University Bern & & 295 & SRR5429446 \\
\hline JF2697 & 2002 & Brazil & Bovine & Vetsuisse University Bern & & 165 & SRR5429445 \\
\hline JF2698 & 2002 & Brazil/unknown & Bovine & Vetsuisse University Bern & & - & - \\
\hline JF3703 & 1956 & New Zealand & Ovine & Wellcome Collection Cf 7 & & 7797 & SRR5429444 \\
\hline JF3837 & 1965 & Switzerland & Bovine & Vetsuisse University Bern & University Bern, Thesis J. Martig & 75 & SRR5429443 \\
\hline JF3838 & 1965 & Switzerland & Bovine & Vetsuisse University Bern & University Bern, Thesis J. Martig & 105 & SRR5429442 \\
\hline JF3840 & 1965 & Switzerland & Bovine & Vetsuisse University Bern & University Bern, Thesis J. Martig & 117 & SRR5429441 \\
\hline JF3841 & 1965 & Switzerland & Bovine & Vetsuisse University Bern & University Bern, Thesis J. Martig & 131 & SRR5429440 \\
\hline JF4057 & 2007 & Switzerland & Bovine & Vetsuisse University Bern & & 101 & SRR5429439 \\
\hline JF4251 & 2002 & Sweden & Bovine & SLU Uppsala & Vilei et al., 2011 & 95 & SRR5429438 \\
\hline JF4252 & 2006 & Sweden & Bovine & SLU Uppsala & Vilei et al., 2011 & 145 & SRR5429437 \\
\hline JF4253 & 2007 & Sweden & Bovine & SLU Uppsala & Vilei et al., 2011 & 141 & SRR5429436 \\
\hline JF4335 & 2004 & Switzerland & Bovine & Vetsuisse University Bern & Falquet et al., 2013 & 34 & SRR5429435 \\
\hline JF4491 & 1956 & New Zealand & Ovine & Wellcome collection Cf 7 & & 8495 & SRR5429434 \\
\hline JF4492 & 1959 & United States & Bovine & Vetsuisse University Bern & & 244 & SRR5429433 \\
\hline JF4493 & 1959 & Australia & Bovine & Wellcome collection Cf 84 & & 7991 & SRR5429432 \\
\hline JF4494 & 1963 & New Zealand & Unknown & Wellcome collection Cf 1 & & 8515 & SRR5429431 \\
\hline JF4495 & 1953 & United Kingdom & Bovine & Wellcome collection Cf44 & Frey et al., 2012 & 8520 & SRR5429430 \\
\hline JF5504 & 2013 & Switzerland & Bovine & Vetsuisse University Bern & & - & - \\
\hline JF5806 & 1951 & South Africa & Ovine & NCTC 8361 & & 248 & SRR5429429 \\
\hline JF5842 & 2015 & Brazil & Bovine & UFSM Jaguari SB 43/15 & R. Ziech, UFSM Brazil & 272 & SRR5429428 \\
\hline
\end{tabular}

Note that strains JF2698 and JF5504 were only used for Figure 1.

using Bowtie2 v2.2.4 with default settings (Langmead et al., 2009). The variant calling was performed for each alignment using the Samtools package v1.3 (Li et al., 2009). All SNVs in the VCF tabular files were concatenated into a FASTA alignment ${ }^{1}$. As a control group Illumina HiSeq 2500 reads from C. septicum (SRA: DRP001713) were used and submitted to the same procedure.

In a previous work a cryptic plasmid was identified (Frey and Falquet, 2015). To find this cryptic plasmid the contigs from the de novo assembly were mapped against the reference PacBio sequence of JF4335 using Bowtie2 v2.2.4 with default settings. Additionally, the raw reads of all 20 strains were assembled with plasmidSPAdes (Antipov et al., 2016).

\section{Phylogenetic Analysis}

The genomes were assembled at the ithree institute (University of Technology Sydney, Australia) using an in-house bioinformatics genome assembly and analysis pipeline (Darling et al., 2014a). Briefly, genomes were assembled using the A5-miseq pipeline (Tritt et al., 2012; Coil et al., 2015), which can process reads up to 500nt long and constructs de Bruijn graphs with $\mathbf{k}$-mers up to 500 nt.

The initial phylogenetic tree was constructed using PhyloSift (Darling et al., 2014b). PhyloSift works by identifying homologs of universally conserved 37 single copy elite markers that are not dependent on operational taxonomic units (OTU; which account for approximately $1 \%$ of an Escherichia coli genome) from any given draft bacterial genome. These include 30 ribosomal protein

\footnotetext{
${ }^{1}$ Bergey CM (2012). vcf-tab-to-fasta; http://code.google.com/p/vcf-tab-to-fasta
}

genes and 7 genes encoding proteins of the translation initiation, elongation and termination process (Darling et al., 2014b). For the purposes of this study, PhyloSift was used to create a concatenated multiple alignment of the marker genes from all our draft genomes. Thirteen C. perfringens genomes from GenBank were included as outgroups. From this alignment, a phylogeny was inferred using FastTree2 (Price et al., 2010) and the resulting tree including the confidence scores (Darling et al., 2014b) was visualized using FigTree v1.4.0 (Figure 1). Internal tree branches were annotated with the support value for each of the clades.

In addition to this explorative approach, all strains were checked for the presence of known genes involved in pathogenicity. Specifically, the putative coding regions for the gene encoding the cytotoxin CctA, hyaluronidases $\mathrm{NagH}$ and NagI, and sialidase NanA were analyzed in detail by mapping the respective gene to the de novo contigs of each strain and by that extracting the corresponding open reading-frame (ORF) from the strains. To assess sequence similarity, we performed a multi-sequence alignment with the translations of the extracted ORFs using MAFFT v7.222 (Katoh et al., 2002; Scoring Matrix: 200PAM $/ k=2$ ).

\section{Analysis of Single Nucleotide Variations (SNV)}

Based on the FASTA alignment of the SNVs a phylogenetic tree was created using the RAxML (options: -m GTRCAT -f d -N

\footnotetext{
${ }^{2}$ Rambaut A.; http://tree.bio.ed.ac.uk/software/figtree/
} 


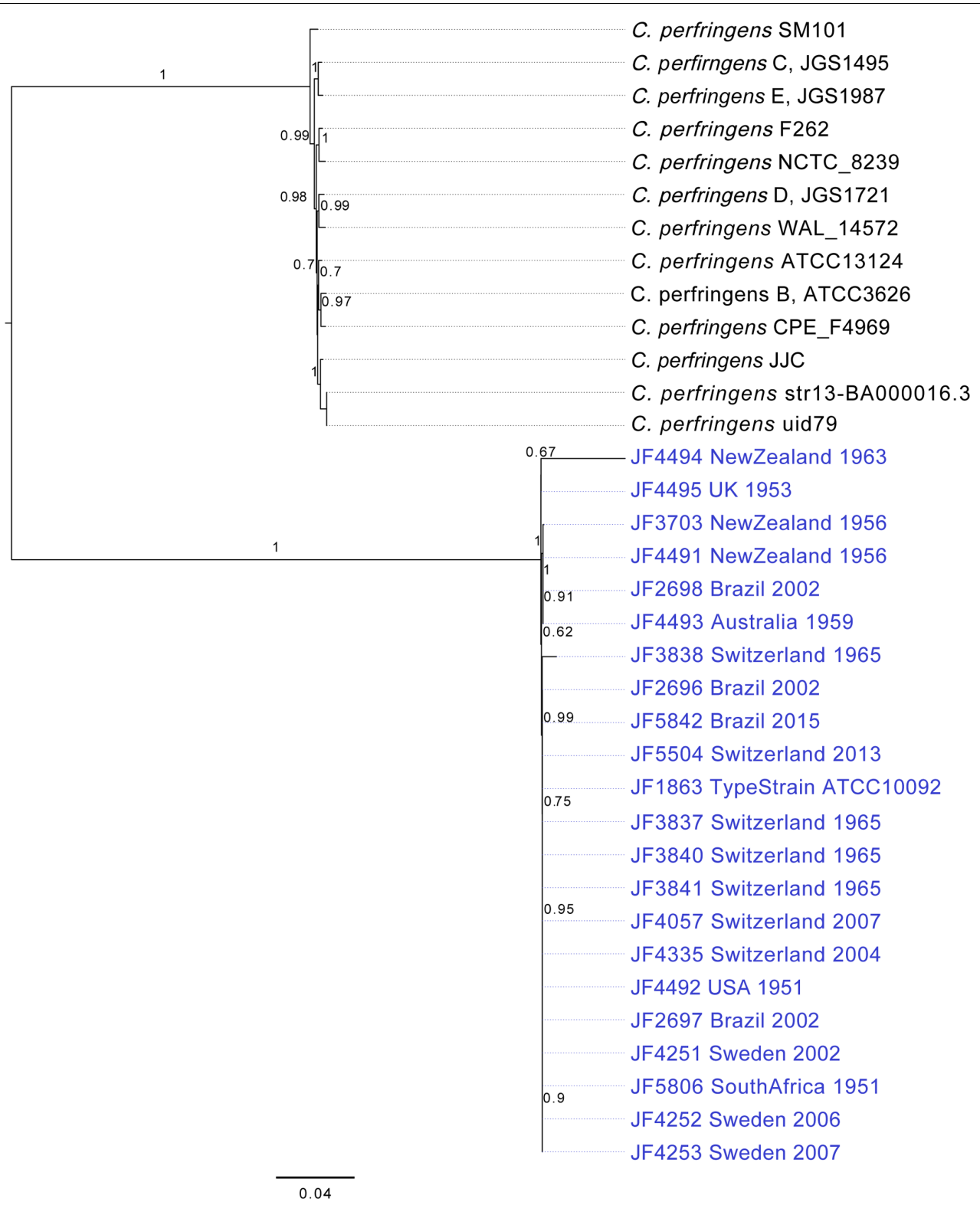

FIGURE 1 | Phylogenetic Trees of the 20 C. chauvoei strains; tree generated with the alignment of concatenated sequence of 37 marker genes searched from the genome of $C$. chauvoei and $C$. perfringens strains whose full genome sequences are publicly accessible, using the PhyloSift software. The bar in the figure indicates substitutions per site. Confidence values are given at the major branching points.

$10^{\prime} 000$-d) (Stamatakis, 2014). Bootstrap values were calculated using RAxML using 10 '000 simulations. The tree was visualized and rooted at the outgroup C. septicum with FigTree v1.4.3. In addition to the phylogenetic tree, a cladogram was created in FigTree.

\section{CRISPR Extraction and Comparison}

To predict the Clustered Regularly Interspaced Short Palindromic Repeats (CRISPR) sequences, the Illumina reads were de novo assembled using SPAdes v3.9.0 (Nurk et al., 2013).

${ }^{3}$ Rambaut A.; http://tree.bio.ed.ac.uk/software/figtree/
The resulting contigs were uploaded to CRISPRFinder (Grissa et al., 2007) for CRISPR prediction. The spacer sequences were oriented within the contigs of each strain based on the CRISPRassociated endoribonuclease 2 (cas2) gene of the reference strain JF4335. Spacer sequences were compared, within and between strains, with each other to find overlapping or unique spacer sequences. They were furthermore compared, within and between strains. Each different spacer sequence was defined as unique, since it was shown that a single nucleotide change in a spacer sequence alters its specificity (Barrangou et al., 2007). For this step NUCmer v3.06 (Kurtz et al., 2004; options: -maxmatch -c 18 -g 3 -1 10) was used. 


\section{RESULTS}

\section{Full Genome Analysis of C. chauvoei Reference Strain JF4335}

Clostridium chauvoei strain JF4335, an isolate from a severe case of blackleg in Switzerland in 2004 was used as reference strain and as reference genome sequence for the species. The genome of this strain was sequenced using Pacific Biosciences R.S. (PacBio) sequencing (85,000 reads/2950 bp average read length). The reads of the PacBio sequencing were assembled with the SMRT Pipeline HGAP3 into one circularized closed contig. The chromosome sequence of C. chauvoei strain JF4335 is $2,887,451$ bp with a G+C content of $28.3 \%$ and with a total of 2624 predicted coding sequences and 114 predicted structural RNAs including transfer tRNAs and ribosomal rRNAs. The complete, circularized and annotated genome was submitted to the European Nucleotide Archive (ENA) database (AC: LT799839). The PacBio sequence also revealed DNA methylations motifs of Restriction Methylation Systems (RMS): GTATA $^{\mathrm{m}}$ C, CCAA $^{\mathrm{m}} \mathrm{N}_{7}$ TTC/GAA ${ }^{\mathrm{m}} \mathrm{N}_{7}$ TTGG and GNGAA ${ }^{\mathrm{m}}$ AY where " $\mathrm{A}$ " is the methylated base m6A. The first motif is a type II RMS (locus_tag = CCH01_18200), the second is a type I RMS (locus_tag = CCH01_22110) and the third was not identified.

\section{Phylogenetic Analysis of C. chauvoei Strains Collected World Wide}

A thorough phylogenetic analysis of the 20 strains, based on a set of 37 OTU-free marker gene families with largely congruent phylogenetic histories revealed hardly any differences between the $C$. chauvoei strains originating from 4 different continents and collected over a period of 64 years. This in contrast to a sample of $C$. perfringens strains whose genomic sequences are published and which originate mostly from Europe and the United States isolated during the last few years (Figure 1). This indicates that C. chauvoei experienced virtually no genetic evolution in the observation period, compared to C. perfringens.

\section{Read Mapping and Consensus Comparison of the 20 International C. chauvoei Strains}

To get an overview of the genomes of the $20 \mathrm{C}$. chauvoei strains, the consensus sequences were extracted from the BAM files using SAMTools and visualized using the BLAST Ring Image Generator (BRIG) (Alikhan et al., 2011) (Upper Threshold 95\%; Lower Threshold 90\%) (Figure 2). A confirmation of the assumed, high similarity between the strains is apparent. The most prevalent gaps in Figure $\mathbf{2}$ were identified and analyzed in more detail within the reference genome. The corresponding DNA segments of these gaps represented mostly fragments of transposases or fragments of putative bacteriophage genes. None of these gaps or islands contains any genes that would be known to have a vital function or to be involved in virulence or pathogenicity of the organism and therefore were not considered any further in this study.
The gene for the major virulence factor, cytotoxin CctA, was found to be present on the chromosome in all strains with an overall nucleotide identity of $99.6 \%$ (Figure 3A). In most strains, the $\operatorname{cctA}$ gene was identical to the reference strain JF4335. However, strains JF3703, JF4491 and JF4493 originating from New Zealand and Australia showed 3 single nucleotide variations (SNVs) representing silent mutations that lead to no difference in the amino acid (a.a.) sequences. Strains JF4494 (New Zealand) and JF4495 (United Kingdom) both revealed the four same SNVs of which one lead to a change in the a.a. sequence of the protein toxin changing the polar neutral glycine at position 167 to the basic a.a. arginine.

All strains contained two hyaluronidase genes nagH and nagI on the chromosome (Figures $\mathbf{3 B}, \mathbf{C}$ ) as found in the reference strain whereof nagH shows a pairwise identity of 99.7\% (max. 42 SNVs out of $5661 \mathrm{bp}$ ) and gene nagI a pairwise identity of $99.1 \%$ (max. 4 SNVs out of 4005 bp) among the strains analyzed. The lower identity of nagI is mainly due to a 340 bp shorter ORF in strain JF4492. Apart from the slight shortening, the nucleotide sequences are almost identical. Minor differences were found in the strains from Australia/New Zealand/United Kingdom cluster, which revealed two SNVs in strains JF3703, JF4491 and JF4493, and three SNVs in JF4494 and JF4495. From these changes one leads to a change in a.a. valine to alanine at position 32 in all five strains and one in strains JF3703, JF4491 and JF4493 at position 115 changing the basic a.a histidine residue to the polar neutral tyrosine.

The gene for the predicted CDS of the sialidase (nanA) (Figure 3D) was found on the chromosome in all strains and showed a $99.0 \%$ pairwise identity (max. 47 SNVs out of 3900 pb). Most notable is a short deletion of 36 bases at the $3^{\prime}$ end of the nucleotide sequence of nanA in the strains from the Australian/New Zealand/United Kingdom cluster. Overall, the virulence genes encoding the main toxin CctA, hyaluronidases $\mathrm{NagH}$ and NagI and sialidase NanA are mostly identical or share a very high similarity in between strains. These similarities are even more pronounced in strains originating from the same country or continent. It is very apparent, that the group of strains originating from Australia, New Zealand and United Kingdom strikingly differs from the strains of the other European countries, Africa, and the Americas. Within the Australian/New Zealand/United Kingdom cluster, we see a more diverse pattern of SNVs, which permits to distinguish them further into subgroup A (JF3703, JF4491 and JF4493) and subgroup B (JF4494 and JF4995) as shown in Figure 3.

To investigate these different groups and attempting to differentiate those further, a single nucleotide variant (SNV) analysis and an analysis of the variable parts (spacers) of the CRISPR locus that was discovered within this study, was performed.

Using plasmidSPAdes analysis, we found a single circular contig of $4.1 \mathrm{kbp}$ in all strains of the European/African/American cluster which corresponded to the same contig that did not get mapped to the chromosome of the reference strain JF4335. This circular contig, shares 95\% identity with the cryptic plasmid previously described (Accession nr: HG323816.1) (Frey and 


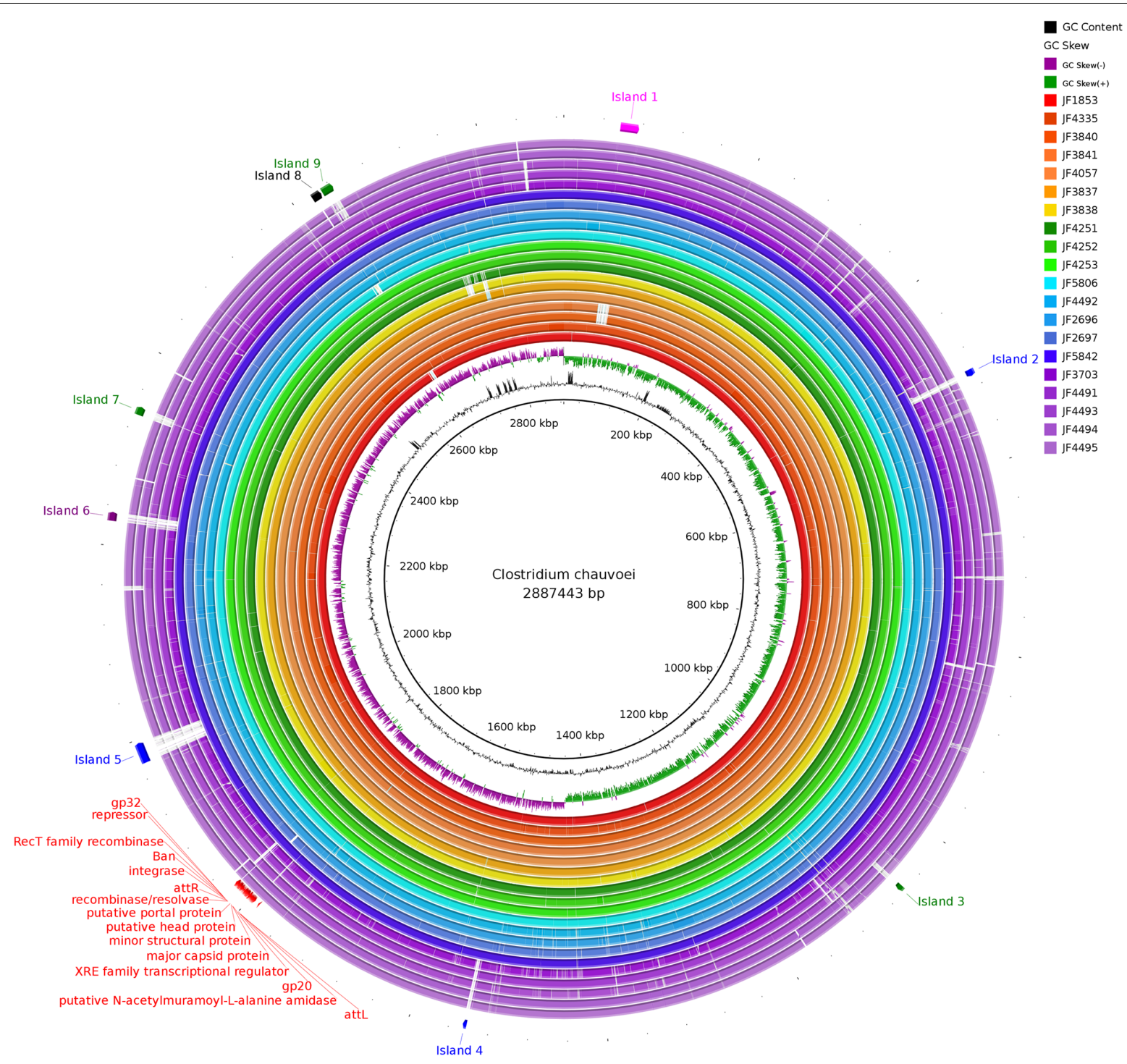

FIGURE 2 | Consensus sequences of all strains aligned against the reference Pacific Biosciences sequence of JF4335 using BLAST Ring Image Generator. Most prevalent gaps (labeled as island 1-9) were inspected for genes potentially involved in virulence or pathogenicity. None of these genes were regarded as such. The gaps mostly represent fragments of transposases and fragments of phage DNA. In red the phages of JF4335, identified with PHASTER (Arndt et al., 2016), are indicated.

Falquet, 2015). As previously noticed these plasmids contain only 4 very short ORF of $150-180 \mathrm{bp}$ and contain no virulence genes.

\section{Single Nucleotide Variations (SNV) Analysis}

In the phylogenetic analysis based on 37 OTU-free marker gene families we could identify virtually no difference between the different $C$. chauvoei strains. To create a better distinction between the twenty strains of $C$. chauvoei, we characterized them by whole genome SNV analysis. Paired-end sequencing was performed using the Illumina technology, yielding 0.6 and 4 million reads per strain. The filtered reads were mapped to the complete genome reference of $C$. chauvoei strain JF4335 obtained by PacBio sequencing. A total of 14,624 total chromosomal SNVs were identified among the worldwide collection of C. chauvoei strains. The numbers of SNVs vary between strains from 34, in the reference strain JF4335, to 8520, within the strain JF4495 from the United Kingdom. Out of the remaining 34 variants in the reference strain JF4335 only 13 were not flagged as low quality by the Samtools filtering and are either short INDELs or SNVs with an unclear resolution that were therefore not corrected after the assembly of the reference sequence with PacBio. The number of SNVs for each strain is given in Table 1. Using the determined SNVs of all strains we created an alignment and built a more detailed phylogenetic tree based on the generalized time reversible substitution model in RAxML. In the resulting tree (Figure 4A) we can confirm the clustering of the strains isolated 


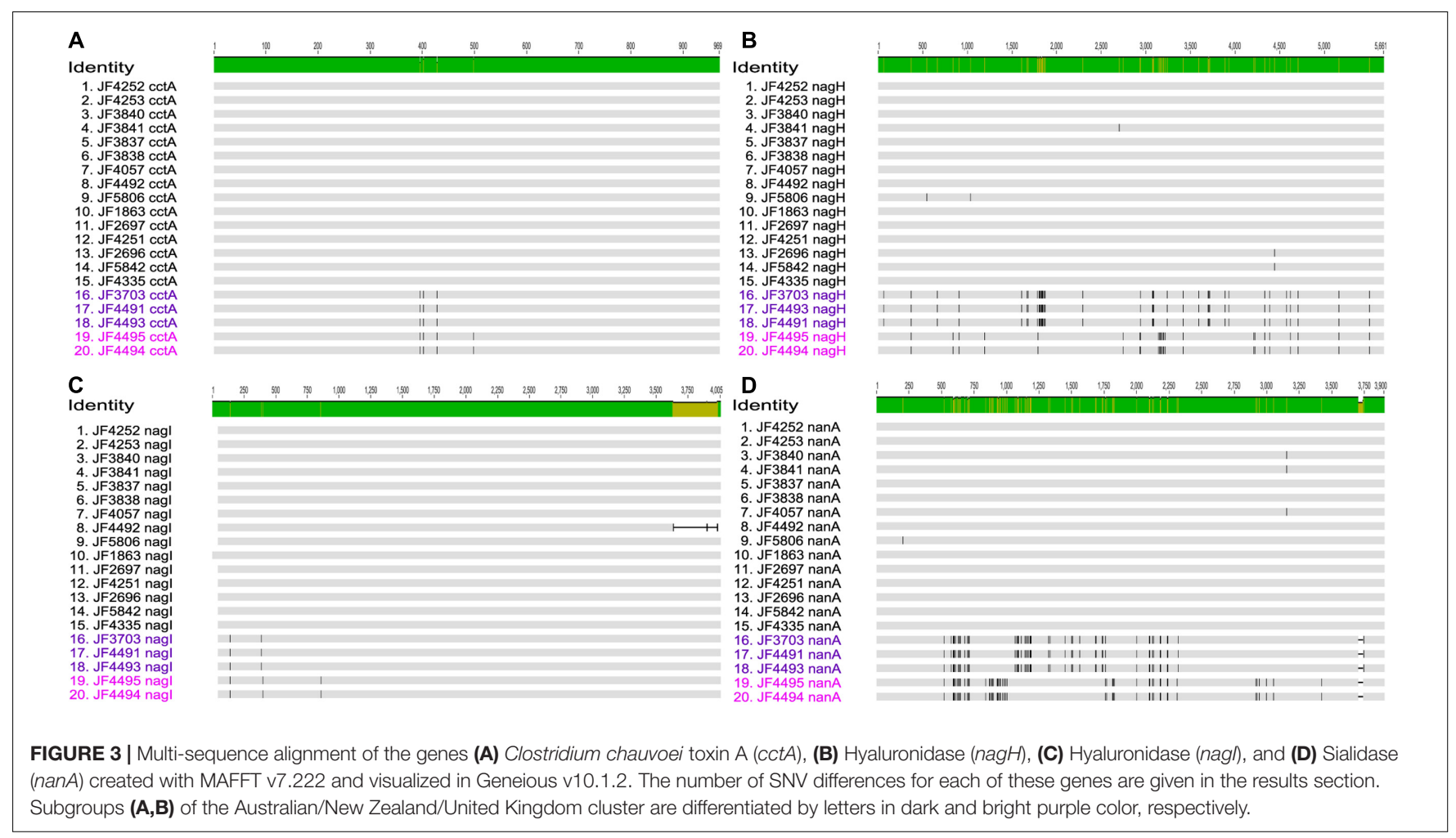

in the New Zealand, Australia and the United Kingdom which is set apart from the remaining strains isolated from Brazil, South Africa, United States, Switzerland and Sweden, independent on the year of isolation. However, since the differences were too small between several strains they appear grouped on the SNV based evolutionary tree. The cladogram shown in Figure 4B allows the visualization of the separation of each strain.

\section{CRISPR Sequences}

The CRISPR sequences found with CRISPRFinder (Grissa et al., 2007) were analyzed for overlapping CRISPR sequences within the genomes of the individual strains and CRISPR sequences among the 20 strains analyzed. We found between 30 and 77 CRISPR sequences per strain in our collection. All CRISPR sequences contain identical interspaced direct repeats (DR) of 30bp length ( $5^{\prime}$-GATTAACATTAACATGAGATGTATTTAAAT$\left.3^{\prime}\right)$. In total, we discovered 1043 CRISPR spacer sequences in the strains analyzed. To individualize spacer sequences within our complete strain collection and identical spacer sequences between the individual strains, we applied an all-vs.-all alignment using NUCmer and an in-house R script to sort them into groups. After both these steps, we got 187 unique spacer sequences and numbered them individually (Figure 5). All spacer sequences are represented in $5^{\prime}$ to $3^{\prime}$ order of appearance downstream of cas 2 in the respective genome (Figure 5). For reasons of visual representation, we aligned identical CRISPRs underneath each other wherever possible. The resulting analysis shows a clustering of the C. chauvoei strains into a European/African/American cluster and an Australia/New Zealand/United Kingdom cluster. This clustering corresponds to that found in the comparison taking into account the four virulence genes cctA, nagI, nagH and nanA or the whole genome SNV analysis, thus confirming the phylogenetic clustering that we found with the two latter approaches.

The spacer sequence analysis of the CRISPR locus of the various C. chauvoei strains reveals that strains belonging to the Australia/New Zealand/United Kingdom cluster strongly and differ from all other strains since they share barely any of the spacer sequences with the strains from the cluster Africa/Europe/America. Furthermore, CRISPR analysis of the Australia/New Zealand/United Kingdom cluster reveals a clear subdivision leading to subgroups A (containing a strain from Australia and two from New Zealand) and subgroup B (a strain from each New Zealand and United Kingdom), which are distinctly different from each other and share no spacers among each other (Figure 5).

A striking feature found in the CRISPR locus of most strains, particularly the strains for the Africa/Europe/America cluster is the presence of a transposase belonging the IS256 family of insertion sequence (IS) in the middle of the repeats splitting the CRISPRs into two arrays (Figure 5; pink box). This transposase is present in all but one strain of the Africa/Europe/American cluster and in subgroup B (New Zealand/United Kingdom) but is not present in subgroup A of the Australia/New Zealand strains. The sequence identity between the IS256 transposase between different strains is $\geq 99 \%$. Surprisingly this transposase is lacking in a recent strain from Brazil (JF5842) that was isolated in 2015, hence later than the other Brazilian strains, which do contain this transposase. Interestingly, in strain JF5842 not only the IS256 transposase, but also the CRISPR spacers \#32 and \#33 upstream 


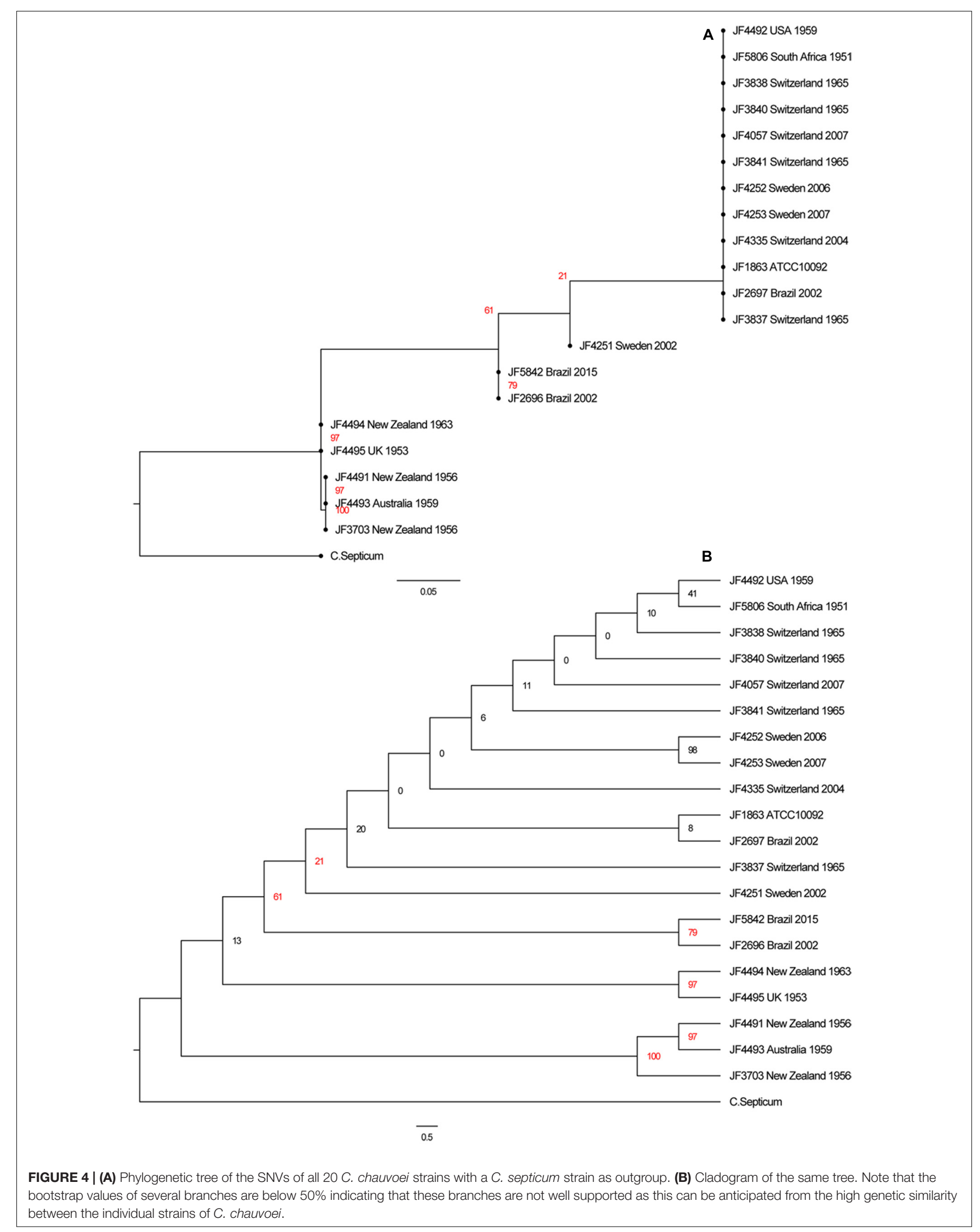




\section{CRISPR-Sequences}

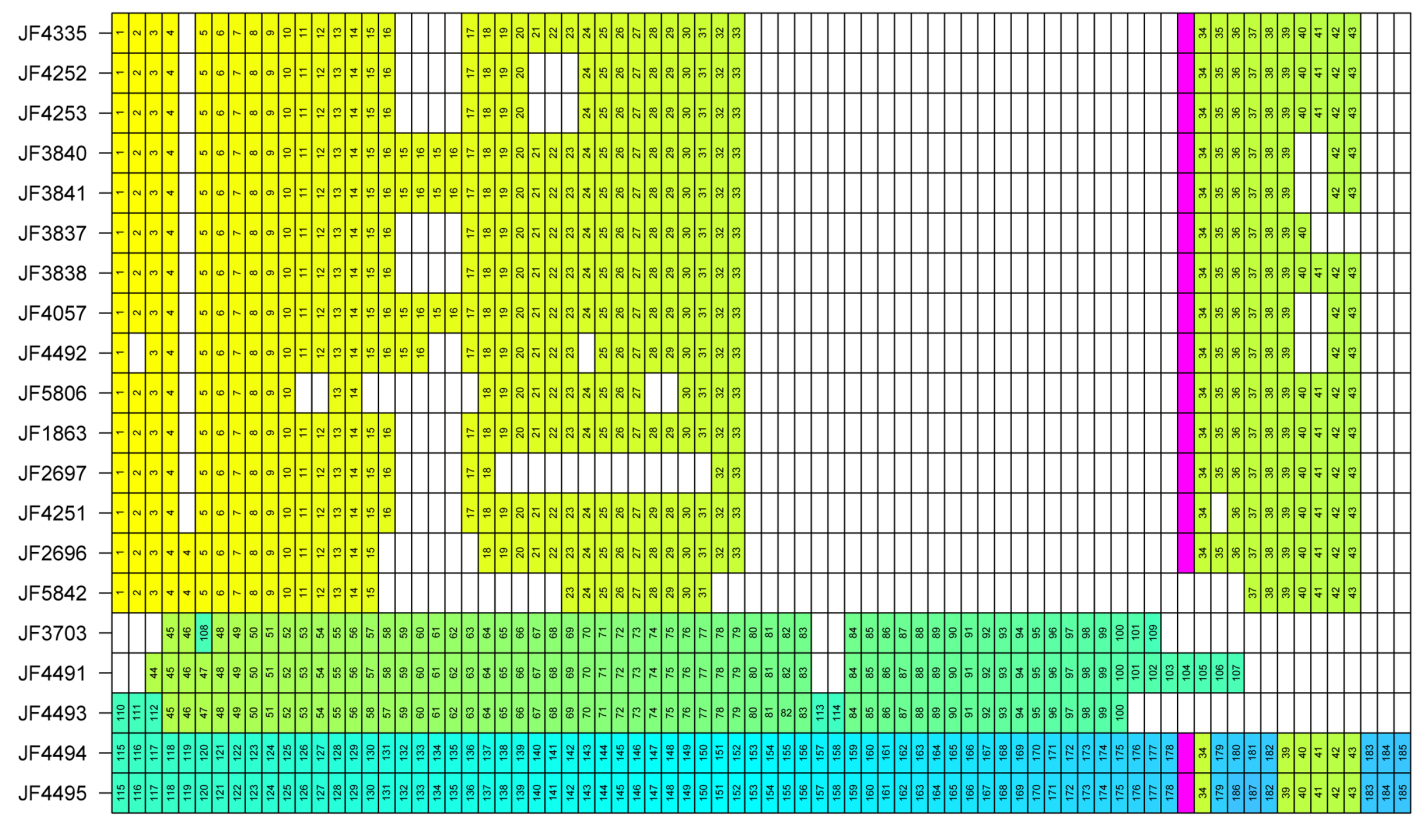

FIGURE 5 | Matrix representation of CRISPR spacers for each individual C. chauvoei strain. Identical spacers are assigned the same numerical value and color. The IS256 transposase is indicated with in pink and divides the CRISPR sequences into two arrays. White spaces are artificial due to the visual representation lining up identical CRISPR spacer sequences and do not represent DNA sequences of the individual genomes. The order of the strains, from top to bottom was arranged to follow clusters and subgroups of the phylogenetic tree of the SNV analysis (Figure 3).

and \#34, \#35 and \#36 downstream of the transposase are lacking in comparison to the closest relative strain JF2696 (Brazil 2002). Furthermore, the presence of the IS256 transposase within the African/European/American cluster and in subgroup B of the New Zealand/United Kingdom strains is particularly interesting as it is lacking in subgroup A of this latter cluster. Moreover, the transposase in the New Zealand and United Kingdom strains is followed at its $3^{\prime}$-end by spacer element \#34 that is common to all the strains of the African/European/American cluster except the recent Brazilian strain discussed above.

Overall, the analysis of the spacer sequences of the CRISPR locus among strains allows a very clear differentiation of most individual strains of $C$. chauvoei and estimate their recent parentage, which by other means such as SNV analysis result in much less resolution power due to the particularly high genetic homology of $C$. chauvoei strains both over space and time. Eighteen isolates are from bovine, three from ovine and one without known animal species. They do, however, not cluster according to the animal species isolated from independently if clustering as done by CRISPR typing or SNV based grouping.

\section{Comparison of Flagellin Genes in Different Strain Groups}

The reference strain JF4335 contains a prominent cluster of three putative genes $f l i C 1, f l i C 2$ and $f l i C 3$, in consecutive order, encoding three different paralog alleles of the structural flagellin proteins. Based on nucleotide-derived a.a. sequences the three flagellin paralogs show $91.8 \%$ a.a. identity among each other.
The read mapping of strains revealed that in particular the strains from subgroup B (JF4494 New Zealand and JF4495 United Kingdom) show very low coverage in the read mapping in the region of the structural flagellin genes indicating that these strains possess fewer alleles of flagellin genes.

To further investigate the flagellin genes in several strains, the reference flagellin genes plus the flanking genes were mapped to the contigs of each strain. The genes flanking the fliC1, fliC2, fliC3 locus were annotated as the flagellar cap protein $\mathrm{D}$, gene $f l i D$, plus a short hypothetical protein upstream and gene $f l i B$, encoding a putative lysine-N-methylase downstream of fliC3 in the reference strain JF4335. As expected, mapping of the contigs primarily revealed a difference in copy number of the structural flagellin genes between $f l i D$ and $f l i B$ or homologs of them. Strains JF4494 (New Zealand) and JF4495 (United Kingdom) that form subgroup B, both only show a single copy of a structural flagellin gene referred to as $f l C 1$ which results in 86 to $87 \%$ identical a.a. residues compared to FliC1, FliC2, FliC3 of reference strain JF4335. This difference of copies of $\mathrm{fliC}$ alleles was confirmed by PCR analysis (data not shown) using primers FLIDL ( $5^{\prime}$-CTAAGCTTGAAGTTGCAATGAAC- $3^{\prime}$ ) and FLIBR $\left(5^{\prime}\right.$-GAATGTGAAGATCATTGCTGTAA- $\left.3^{\prime}\right)$, which resulted in a fragment of $1.7 \mathrm{~kb}$ for strain JF4494 with one copy and a fragment of $5 \mathrm{~kb}$ for strains with 3 copies of $\mathrm{fliC}$ alleles, such as the reference strain JF4335. The Swedish strain JF4252 and the United States strain JF4492 show two copies of structural flagellin genes. In JF4252 (Sweden) one gene showed full homology to the FliC2 and the other $99.8 \%$ identical a.a. to FliC1 of JF4335. 
TABLE 2 | Pair-wise identities of all the FliC genes in the strains JF4335, JF4252, JF4492, JF4494, and JF4495 created by a MAFFT multi-sequence alignment.

\begin{tabular}{|c|c|c|c|c|c|c|c|c|c|}
\hline Nucleotide & JF4335 fliC1 & JF4335 fliC2 & JF4335 fliC3 & JF4252 fliC1 & JF4252 fliC2 & JF4492 fliC1 & JF4492 fliC2 & JF4494 fliC1 & JF4495 fliC1 \\
\hline JF4335 fliC1 & 1 & 0.931 & 0.931 & 0.928 & 0.997 & 0.931 & 0.964 & 0.866 & 0.866 \\
\hline JF4335 fliC2 & & 1 & 0.969 & 0.997 & 0.934 & 0.969 & 0.967 & 0.868 & 0.868 \\
\hline JF4335 flic3 & & & 1 & 0.972 & 0.934 & 1 & 0.936 & 0.861 & 0.861 \\
\hline JF4252 fliC1 & & & & 1 & 0.931 & 0.972 & 0.964 & 0.87 & 0.87 \\
\hline JF4252 fliC2 & & & & & 1 & 0.934 & 0.967 & 0.864 & 0.864 \\
\hline JF4492 fliC1 & & & & & & 1 & 0.936 & 0.861 & 0.861 \\
\hline JF4492 fliC2 & & & & & & & 1 & 0.872 & 0.872 \\
\hline JF4494 fliC1 & & & & & & & & 1 & 1 \\
\hline JF4495 fliC1 & & & & & & & & & 1 \\
\hline Protein & JF4335 FliC1 & JF4335 FliC2 & JF4335 FliC3 & JF4252 FliC1 & JF4252 FliC2 & JF4492 FliC1 & JF4492 FliC2 & JF4494 FliC1 & JF4495 FliC1 \\
\hline JF4335 FliC1 & 1 & 0.918 & 0.918 & 0.918 & 0.998 & 0.918 & 0.961 & 0.826 & 0.826 \\
\hline JF4335 FliC2 & & 1 & 0.966 & 1 & 0.92 & 0.966 & 0.956 & 0.835 & 0.835 \\
\hline JF4335 FliC3 & & & 1 & 0.966 & 0.92 & 1 & 0.923 & 0.823 & 0.823 \\
\hline JF4252 FliC1 & & & & 1 & 0.92 & 0.966 & 0.956 & 0.835 & 0.835 \\
\hline JF4252 FliC2 & & & & & 1 & 0.92 & 0.964 & 0.823 & 0.823 \\
\hline JF4492 FliC1 & & & & & & 1 & 0.923 & 0.823 & 0.823 \\
\hline JF4492 FliC2 & & & & & & & 1 & 0.838 & 0.838 \\
\hline JF4494 FliC1 & & & & & & & & 1 & 1 \\
\hline JF4495 FliC1 & & & & & & & & & 1 \\
\hline
\end{tabular}

In a multi-sequence alignment on the ORFs, they have a $92.1 \%$ pairwise nucleotides identity and a $90.3 \%$ pairwise a.a. identity among each other. Similar results were found for the United States strain JF4492 that has one of its structural flagellin genes homologous to FliC3 and one with $96.1 \%$ a.a. identity to FliC1 of reference strain JF4335. A complete table of all nucleotide and a.a. similarities between the various structural flagellin genes is given in Table 2.

Interestingly, the 413 amino-acid long sequences of FliC1, FliC2 and FliC3 among themselves and among the various strains analyzed show their variability between position 174 and 302, whereas the $\mathrm{N}$ - and $\mathrm{C}$-termini are mostly conserved (Figure 6). We detect a putative conserved domain described as flagellin D0/D1 domain (IPR001029) in all structural flagellin genes in all strains analyzed. This can lead to the assumption that they fulfill a similar task in all strains. However, this central hyper variable region could be of critical importance when designing recombinant vaccines using flagellin proteins.

\section{DISCUSSION}

Blackleg is a global, severe, and mostly lethal bacterial infectious disease of ruminants caused by the sporulating bacterium C. chauvoei. Due to its rapid progression, there are hardly any therapies for the affected individuals. In contrast, preventive measures by vaccination with empirically developed bacterin vaccines are efficient and protect animals grazing on meadows that are contaminated by spores of C. chauvoei. In spite of the worldwide spread of blackleg, there are hardly any studies published on the molecular mechanisms of pathogenicity and epidemiological spread of the organism. These, however, would be of high importance in the design and composition of vaccines and testing their efficacies. Interestingly, international bacterial strain collections conserve hardly any clinical strains of C. chauvoei, besides the type strain whose exact origin and date of isolation is unknown. We therefore performed full genome sequencing and bioinformatics analysis of a collection of 20 strains of $C$. chauvoei isolated from bovine and ovine sources over the last 64 years on four different continents that were maintained at the internal strain collection of the Institute of Veterinary Bacteriology, University of Bern. The Swiss strains were isolated in the past from clinical cases of blackleg in cattle. The other strains were either purchased from international strain collections or obtained from other laboratories for purpose of species identification. In spite of vast temporal and geographical diversity of these strains, the phylogenetic analysis based on 37 OTU-free marker gene families of the different $C$. chauvoei strains showed virtually no genetic variations and behaved like a single strain. In addition, the analysis of the major virulence genes such as the main cytotoxin CctA, the sialidase NanA and the two hyaluronidases NagI, NagH show hardly any variations on the a.a. level indicating identical phenotypes. Hence, the evolution of C. chauvoei seems to have stopped from the point of view of metabolism and virulence factors and the pathogen seems to be well adapted to its bimodal way of life using as host, cattle and sheep, where it replicates and the environment, the meadows, where it persists as spores.

While SNVs, which mostly are located in intergenic segments of the chromosome, allow a finer differentiation of the strains, the spread of the pathogen can be followed by the large number of CRISPR elements. While the CRISPR associated protein genes in all strains are well conserved, we detected in total 187 unique spacer sequences within the CRISPR elements that 


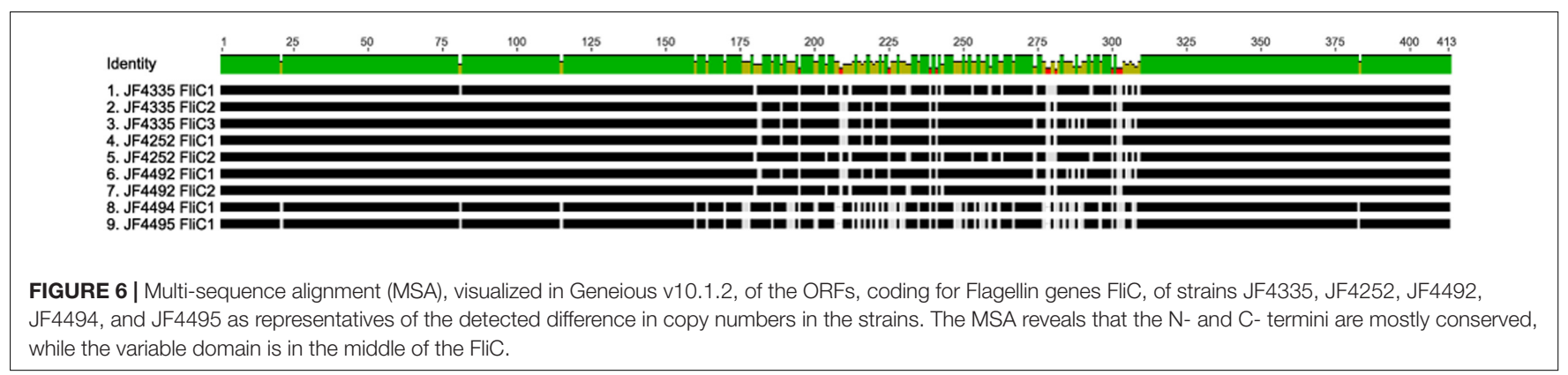

are reminiscences of successfully warded phage attacks. These show significant differences between the strains from Africa, continental Europe and Northern and Southern America and strains from United Kingdom, Australia and New Zealand. There are surprisingly very little differences found between strains from Switzerland, Sweden, Brazil and South Africa, which contain virtually identical spacer sequences throughout the 40 different CRISPR elements. The presence of an insertion element from the IS256 family, inserted after approximately the 30th spacer element is peculiar. In a recently isolated strain from Brazil from 2015, this insertion element is deleted and, in comparison with the two strains isolated in 2002 the excision of the IS seems to have removed also five flanking CRISPRs. This indicates that this IS element is still active and able to transpose.

The strains from Australia, New Zealand and the United Kingdom reveal totally different spacer sequences, compared to the strains from Switzerland, Sweden, Brazil and South Africa, which reflect the close cultural and agricultural exchange between these countries over a long historical period. This is illustrated with two strains JF4494 isolated in 1963 in New Zealand, which is identical to strain JF4495 isolated 10 years previously in the United Kingdom. The fact that there are two completely different CRISPR sequences found in strains in New Zealand indicates that the pathogen must have been introduced twice in the past, once in connection with Australia and one in connection with the United Kingdom. Interestingly, the strains from the second event (JF4494 and JF4495) not only are identical with regard to the CRISPRs but also show the presence of the IS256 directly followed by CRISPR spacer \#34 which is found in the Africa/Europe/America cluster. Furthermore, both strains also contain five CRISPR spacers (\#39 - \#43), probably as recently acquirements indicating that they had to fend off the same or similar phages as the strains from the Africa/Europe/America cluster.

Since flagella have been considered as important protective antigens, we have put particular attention to the genetic variations of flagellar genes among the strains analyzed. While the basal flagellar genes are mostly identical or show only irrelevant differences among all strains, we detected differences in the flagellin genes $\mathrm{fliC}$. Most importantly, $\mathrm{fliC}$ appears as three allelic copies with $91.8 \%$ identity in most strains, while two strains, one from New Zealand and one from the United Kingdom, only contained a single copy. A strain from Sweden and the one from United States also contained two copies. Since the three alleles of $\mathrm{fliC}$ are contiguous and very similar among each other, the loss of one or two alleles can be explained by homologous recombination events. Furthermore it has to be noted that a single $f$ liC allele in the relatively ancient strain from New Zealand and from the United Kingdom show only in $86 \%$ to $87 \%$ identity to the $f l i C$ alleles that are present in most other strains. If the FliC antigens do play a role in protective immunity induced by vaccines, an assessment of the $\mathrm{fliC}$ alleles in currently circulating strains in the areas where vaccines are used would be useful. The current data, in particular the PCR primers developed in this study will provide a useful tool for this purpose.

While the study contains relatively few strains taken from most distant geographical places itn has to be taken into consideration that the data need to be interpreted with precaution with regards to specific countries or animal origins of the strains. It is interesting to note that the few ovine strains included in this study do not correlate to any of the grouping made by CRISPR, SNV or virulence gene typing. Hence, the clusters of C. chauvoei described seem not to show host predilection.

Besides the relatively minor genetic differences found in the flagellar genes and in the CRISPR spacers our analysis reveals that the genome of $C$. chauvoei, which is the smallest among the species of the genus Clostridium, is highly conserved among strains collected from most distant geographical areas and over a long time frame. This indicates that $C$. chauvoei is a highly specialized and successful pathogen whose evolution has reached a dead end.

\section{AUTHOR CONTRIBUTIONS}

JF, LF, and SD conceived the study and performed the genomic analyses. PN performed the strain isolations and DNA preparations. Bioinformatic analyses were made by SI-A and LR. PC established the phylogenic relationship using by PhyloSift. $\mathrm{RZ}$ and AdV provided the strains and metadata from strains of Brazil.

\section{FUNDING}

This research was supported by the Research Foundation 3R (Reduction, Refinement and Replacement of Animal Experimentation) grant no. 136-13 to JF. 


\section{ACKNOWLEDGMENTS}

The sequencing was performed by the Next Generation Sequencing (NGS) Platform of the University of Bern (http://www.ngs.unibe.ch) and by the Genomics Technology Facility of the University of Lausanne (http://www.unil.ch/gtf).

\section{REFERENCES}

Alikhan, N. F., Petty, N. K., Zakour, N. L. B., and Beatson, S. A. (2011). BLAST ring image generator (BRIG): simple prokaryote genome comparisons. BMC Genomics 12:402. doi: 10.1186/1471-2164-12-402

Antipov, D., Hartwick, N., Shen, M., Raiko, M., Lapidus, A., and Pevzner, P. A. (2016). plasmidSPAdes: assembling plasmids from whole genome sequencing data. Bioinformatics 32, 3380-3387. doi: 10.1093/bioinformatics/btw493

Arndt, D., Grant, J. R., Marcu, A., Sajed, T., Pon, A., Liang, Y., et al. (2016). PHASTER: a better, faster version of the PHAST phage search tool. Nucleic Acids Res. 44, W16-W21. doi: 10.1093/nar/gkw387

Bagge, E., Lewerin, S. S., and Johansson, K. E. (2009). Detection and identification by PCR of Clostridium chauvoei in clinical isolates, bovine faeces and substrates from biogas plant. Acta Vet. Scand. 51:8. doi: 10.1186/1751-0147-51-8

Barrangou, R., Fremaux, C., Deveau, H., Richards, M., Boyaval, P., Moineau, S., et al. (2007). CRISPR provides acquired resistance against viruses in prokaryotes. Science 315, 1709-1712. doi: 10.1126/science.1138140

Chandler, H. M., and Gulasekharam, J. (1974). The protective antigen of a highly immunogenic strain of Clostridium chauvoei including an evaluation of its flagella as a protective antigen. Microbiology 84, 128-134. doi: 10.1099/ 00221287-84-1-128

Coil, D., Jospin, G., and Darling, A. E. (2015). A5-miseq: an updated pipeline to assemble microbial genomes from Illumina MiSeq data. Bioinformatics 31, 587-589. doi: 10.1093/bioinformatics/btu661

Darling, A. E., Worden, P., Chapman, T. A., Chowdhury, P. R., Charles, I. G., and Djordjevic, S. P. (2014a). The genome of Clostridium difficile 5.3. Gut Pathog. 6:4. doi: 10.1186/1757-4749-6-4

Darling, A. E., Jospin, G., Lowe, E., Matsen, F. A. I. V., Bik, H. M., and Eisen, J. A. (2014b). PhyloSift: phylogenetic analysis of genomes and metagenomes. PeerJ 2:e243. doi: $10.7717 /$ peerj. 243

Falquet, L., Calderon-Copete, S. P., and Frey, J. (2013). Draft genome sequence of the virulent Clostridium chauvoei reference strain JF4335. Genome Announc. 1:e00593-13. doi: 10.1128/genomeA.00593-13

Frey, J., and Falquet, L. (2015). Patho-genetics of Clostridium chauvoei. Res. Microbiol. 166, 384-392. doi: 10.1016/j.resmic.2014.10.013

Frey, J., Johansson, A., Bürki, S., Vilei, E. M., and Redhead, K. (2012). Cytotoxin CctA, a major virulence factor of Clostridium chauvoei conferring protective immunity against myonecrosis. Vaccine 30, 5500-5505. doi: 10.1016/j.vaccine. 2012.06.050

Grissa, I., Vergnaud, G., and Pourcel, C. (2007). CRISPRFinder: a web tool to identify clustered regularly interspaced short palindromic repeats. Nucleic Acids Res. 35(Suppl. 2), W52-W57. doi: 10.1093/nar/gkm360

Groseth, P. K., Ersdal, C., Bjelland, A. M., and Stokstad, M. (2011). Large outbreak of blackleg in housed cattle. Vet. Rec. 169, 339-339. doi: 10.1136/vr.d4628

Hatheway, C. L. (1990). Toxigenic clostridia. Clin. Microbiol. Rev. 3, 66-98. doi: 10.1128/CMR.3.1.66

Jubb, K. V. F., Kennedy, P. C., and Palmer, N. (1991). Pathology of Domestic Animals, 4th Edn. San Diego, CA: Academic Press.

Katoh, K., Misawa, K., Kuma, K. I., and Miyata, T. (2002). MAFFT: a novel method for rapid multiple sequence alignment based on fast Fourier transform. Nucleic Acids Res. 30, 3059-3066. doi: 10.1093/nar/gkf436

Kurtz, S., Phillippy, A., Delcher, A. L., Smoot, M., Shumway, M., Antonescu, C., et al. (2004). Versatile and open software for comparing large genomes. Genome Biol. 5:R12. doi: 10.1186/gb-2004-5-2-r12

Lange, M., Neubauer, H., and Seyboldt, C. (2010). Development and validation of a multiplex real-time PCR for detection of Clostridium chauvoei and Clostridium septicum. Mol. Cell. Probes 24, 204-210. doi: 10.1016/j.mcp.2010.03.003
The computations were performed at the Vital-IT (http://www. vital-it.ch) center for high-performance computing of the Swiss Institute of Bioinformatics and on UBELIX (http://www.id.unibe. $\mathrm{ch} / \mathrm{hpc}$ ), the HPC cluster at the University of Bern. We are grateful to Isabelle Brodard and Alexandra Collaud for their help with PCR analyses.

Langmead, B., Trapnell, C., Pop, M., and Salzberg, S. L. (2009). Ultrafast and memory-efficient alignment of short DNA sequences to the human genome. Genome Biol. 10:R25. doi: 10.1186/gb-2009-10-3-r25

Li, H., Handsaker, B., Wysoker, A., Fennell, T., Ruan, J., Homer, N., et al. (2009). The sequence alignment/map format and SAMtools. Bioinformatics 25, 2078-2079. doi: 10.1093/bioinformatics/btp352

Mattar, M. A., Cortiñas, T. I., and Guzmán, A. M. S. (2002). Immunogenic protein variations of Clostridium chauvoei cellular antigens associated with the culture growth phase. FEMS Immunol. Med. Microbiol. 33, 9-14. doi: 10.1111/j.1574695X.2002.tb00565.x

Nagano, N., Isomine, S., Kato, H., Sasaki, Y., Takahashi, M., Sakaida, K., et al. (2008). Human fulminant gas gangrene caused by Clostridium chauvoei. J. Clin. Microbiol. 46, 1545-1547. doi: 10.1128/JCM.01895-07

Nurk, S., Bankevich, A., Antipov, D., Gurevich, A. A., Korobeynikov, A., Lapidus, A., et al. (2013). Assembling single-cell genomes and minimetagenomes from chimeric MDA products. J. Comput. Biol. 20, 714-737. doi: $10.1089 / \mathrm{cmb} .2013 .0084$

Price, M. N., Dehal, P. S., and Arkin, A. P. (2010). FastTree 2-approximately maximum-likelihood trees for large alignments. PLoS ONE 5:e9490. doi: 10.1371/journal.pone.0009490

Seemann, T. (2014). Prokka: rapid prokaryotic genome annotation. Bioinformatics 30, 2068-2069. doi: 10.1093/bioinformatics/btu153

Stamatakis, A. (2014). RAxML version 8: a tool for phylogenetic analysis and post-analysis of large phylogenies. Bioinformatics 30, 1312-1313. doi: 10.1093/ bioinformatics/btu033

Tamura, Y., Kijima-Tanaka, M., Aoki, A., Ogikubo, Y., and Takahashi, T. (1995). Reversible expression of motility and flagella in Clostridium chauvoei and their relationship to virulence. Microbiology 141, 605-610. doi: 10.1099/13500872141-3-605

Tamura, Y., and Tanaka, M. (1987). Opsonic activity of anti-flagellar serum against Clostridium chauvoei by mouse polymorphonuclear leucocytes. Vet. Microbiol. 14, 81-86. doi: 10.1016/0378-1135(87)90055-1

Tanaka, M., Hirayama, N., and Tamura, Y. (1987). Production, characterization, and protective effect of monoclonal antibodies to Clostridium chauvoei flagella. Infect. Immun. 55, 1779-1783.

Tritt, A., Eisen, J. A., Facciotti, M. T., and Darling, A. E. (2012). An integrated pipeline for de novo assembly of microbial genomes. PLoS ONE 7:e42304. doi: 10.1371/journal.pone.0042304

Useh, N. M., Ajanusi, J. O., Esievo, K. A. N., and Nok, A. J. (2006a). Characterization of a sialidase (neuraminidase) isolated from Clostridium chauvoei (Jakari strain). Cell Biochem. Funct. 24, 347-352.

Useh, N. M., Ibrahim, N. D. G., Nok, A. J., and Esievo, K. A. N. (2006b) Relationship between outbreaks of blackleg in cattle and annual rainfall in Zaria, Nigeria. Vet. Rec. 158, 100-101. doi: 10.1136/vr.158.3.100

Useh, N. M., Nok, A. J., and Esievo, K. A. N. (2003). Pathogenesis and pathology of blackleg in ruminants: the role of toxins and neuraminidase. A short review. Vet. Q. 25, 155-159.

Usharani, J., Nagaleekar, V. K., Thomas, P., Gupta, S. K., Bhure, S. K., Dandapat, P., et al. (2015). Development of a recombinant flagellin based ELISA for the detection of Clostridium chauvoei. Anaerobe 33, 48-54. doi: 10.1016/j.anaerobe. 2015.02.001

Uzal, F. A. (2012). Evidence-based medicine concerning efficacy of vaccination against Clostridium chauvoei infection in cattle. Vet. Clin. N. Am. 28, 71-77. doi: 10.1016/j.cvfa.2011.12.006

Vilei, E. M., Johansson, A., Schlatter, Y., Redhead, K., and Frey, J. (2011). Genetic and functional characterization of the NanA sialidase from Clostridium chauvoei. Vet. Res. 42:2. doi: 10.1186/1297-9716-42-2 
Weatherhead, J. E., and Tweardy, D. J. (2012). Lethal human neutropenic entercolitis caused by Clostridium chauvoei in the United States: tip of the iceberg? J. Infect. 64, 225-227. doi: 10.1016/j.jinf.2011.09.004

Wolf, R., Hiesel, J., Kuchling, S., Deutz, A., Kastelic, J., Barkema, H. W., et al. (2017). Spatial-temporal cluster analysis of fatal Clostridium chauvoei cases among cattle in Styria, Austria between 1986 and 2013. Prevent. Vet. Med. 138, 134-138. doi: 10.1016/j.prevetmed.2017.01.019

Conflict of Interest Statement: The authors declare that the research was conducted in the absence of any commercial or financial relationships that could be construed as a potential conflict of interest. The research was done with the final aim to replace the currently used guinea pig potency test for batch release of blackleg vaccines by an in vitro procedure thus avoiding challenge of animals with pathogens.

Copyright (c) 2017 Rychener, In-Albon, Djordjevic, Chowdhury, Nicholson, Ziech, de Vargas, Frey and Falquet. This is an open-access article distributed under the terms of the Creative Commons Attribution License (CC BY). The use, distribution or reproduction in other forums is permitted, provided the original author(s) or licensor are credited and that the original publication in this journal is cited, in accordance with accepted academic practice. No use, distribution or reproduction is permitted which does not comply with these terms. 